\title{
Reflets
}

Revue d'intervention sociale et communautaire

\section{Campagne du consentement au Yukon}

\section{Sarah Cloutier}

Volume 21, numéro 1, printemps 2015

URI : https://id.erudit.org/iderudit/1032555ar

DOI : https://doi.org/10.7202/1032555ar

Aller au sommaire du numéro

Éditeur(s)

Reflets, Revue d'intervention sociale et communautaire

ISSN

1203-4576 (imprimé)

1712-8498 (numérique)

Découvrir la revue

Citer cet article

Cloutier, S. (2015). Campagne du consentement au Yukon. Reflets, 21(1),

206-214. https://doi.org/10.7202/1032555ar d'utilisation que vous pouvez consulter en ligne.

https://apropos.erudit.org/fr/usagers/politique-dutilisation/ 


\section{Campagne du consentement au Yukon}

\section{Sarah Cloutier}

La violence sexuelle est une problématique de taille auYukon. Le taux d'agressions sexuelles y est 3,5 fois plus élevé que la moyenne des provinces et territoires canadiens (Statistique Canada, 2013). Mais la communauté yukonnaise est loin d'être indifférente à cette triste réalité; nombreuses sont les initiatives visant à contrer cette violence. Entre autres, une association de Franco-Yukonnaises, les EssentiElles, mène depuis quatre ans une campagne de sensibilisation sur le consentement et les agressions sexuelles. Sa Campagne du consentement se tient durant l'été, principalement lors de différents festivals qui se déroulent sur le territoire.

À l'été 2013,j'ai eu la chance de travailler chez les EssentiElles comme coordonnatrice de la campagne. Cela m'a permis de découvrir la communauté franco-yukonnaise et de participer à un modèle intéressant et novateur de prévention de la violence sexuelle. Le présent article porte d'abord sur le féminisme yukonnais tel que je l'ai découvert lors de cette expérience de travail, puis plus spécifiquement sur son implication contre la violence sexuelle. Par la suite, j'y présente plus en détail la Campagne du consentement, en soulignant ses impacts et certains défis rencontrés.

\section{Une implication féministe diversifiée pour une communauté diversifiée}

Dès mon arrivée au Yukon, j'ai constaté que malgré son nombre peu élevé d'habitants (environ 36000 selon le Yukon 
Bureau of Statistics [2012]) l'activisme féminisme y est bien présent et vivant, tant sur le plan individuel qu'à travers certains organismes, dont les EssentiElles. La population du territoire y est aussi diversifiée. Présente au Yukon depuis les années 1800, la communauté francophone y joue un rôle important dans les sphères culturelles et sociales. Les Francophones ont réalisé dans les années 1980 la nécessité de se regrouper pour défendre leur langue et leurs droits. C'est ainsi que l'Association francoyukonnaise a été créée en 1982 dans le but d'assurer le bien-être durable de la collectivité francophone du territoire, notamment par le développement de services et d'activités (Association franco-yukonnaise, 2014). Encore aujourd'hui très active au sein de la communauté yukonnaise, l'association travaille avec des partenaires francophones, comme les EssentiElles, et non francophones. Que ce soit par cette association et ses partenaires ou par d'autres groupes ou individus, les Franco-Yukonnais s'impliquent grandement dans l'ensemble des enjeux sociaux et politiques du territoire.

Avec environ $25 \%$ des habitants du territoire, les Premières Nations (les Tutchones du Nord, les Tutchones du Sud et les Tlingits) occupent elles aussi une place importante au sein de la population duYukon (Gouvernement duYukon, 2008; Statistique Canada, 2012). Leur présence et leur culture y sont valorisées et célébrées, notamment lors de divers événements et festivals. Les communautés autochtones revendiquent leurs droits à travers différents groupes et organisations, tels le Conseil des Premières Nations du Yukon, le Conseil des femmes autochtones du Yukon et le Whitehorse Aboriginal Women's Circle.

Lors de mon séjour, j'ai pu constater que les actions féministes, et en particulier les initiatives contre la violence faite aux femmes, sont souvent mises en place conjointement par des regroupements de femmes anglophones, autochtones et francophones. Dans ce domaine, les Franco-Yukonnaises sont représentées principalement par les EssentiElles, pour qui la lutte contre la violence faite aux femmes constitue l'un des principaux champs d'action. La Campagne du consentement est un exemple de cette collaboration : elle a été mise sur pied par les EssentiElles 
en collaboration avec le Centre pour femmesVictoria Faulkner qui est majoritairement anglophone. La campagne compte également sur le soutien du Kaushee's Place, un centre d'hébergement pour femmes, nommé en l'honneur de Kaushee Harris, une Tlingit leader du droit des femmes dans sa communauté. Plusieurs membres de l'équipe de ce centre sont des femmes autochtones. Cette collaboration dans la Campagne du consentement permet de s'assurer que les actions mises en place représentent bien les intérêts des femmes des différentes collectivités du Yukon. On peut cependant noter l'absence significative d'une représentation de la communauté immigrante, notamment philippine, qui est pourtant importante dans le territoire.

\section{Un changement de direction dans la prévention de la violence sexuelle}

À mes débuts à Whitehorse pour travailler aux EssentiElles, on m'a expliqué qu'au cours des dernières années, la direction prise par les groupes de femmes yukonnaises dans la prévention de la violence sexuelle avait changé. Auparavant, l'accent était mis principalement sur les femmes et les potentielles victimes : quoi faire pour se protéger? Comment aller chercher de l'aide? etc. Les groupes de femmes ont réalisé que d'abord cette approche n'était pas efficace pour contrer la violence sexuelle. Qui plus est, en mettant la responsabilité sur les femmes pour prévenir la violence, elle pouvait également, se retrouver en contradiction avec l'analyse féministe de cette problématique, qui voit la domination masculine comme cause de la violence et qui vise à déresponsabiliser les victimes. De plus, au Canada, $82 \%$ des agressions sexuelles sont commises par une personne connue de la victime (Statistique Canada, 2013). Ainsi, les traditionnelles mises en garde telles que "Ne vous promenez pas seule le soir » ou "Surveillez votre verre au bar» ne semblent pas des plus appropriées.

Pour ces raisons, les initiatives yukonnaises de prévention de la violence sexuelle visent depuis quelques années à développer au sein de la communauté une meilleure compréhension 
du consentement et à éduquer les hommes principalement - puisque $97 \%$ des agressions sexuelles sont commises par des hommes (Statistique Canada, 2008) - sur les moyens de respecter le consentement. Cette position des groupes de femmes yukonnais correspond d'ailleurs à celle adoptée par une grande partie des groupes de femmes à travers le pays. La Campagne du consentement créée par les EssentiElles et ses partenaires s'inscrit donc dans ce nouvel objectif d'une responsabilité sociale commune face à la prévention de la violence et d'une responsabilisation des agresseurs plutôt que celle des victimes.

\section{La sex positivity pour rejoindre les festivaliers}

La Campagne du consentement est née par hasard. En 2010, en collaboration avec le Centre pour femmes Victoria Faulkner, les EssentiElles a fait imprimer des autocollants portant différents messages sur le consentement sexuel, tels que "Consent is sexy " et «Non veut dire non ». Destinés à un public adolescent, ils ont été distribués dans des écoles. La réaction des administrations scolaires a été rapide... et négative : les autocollants avaient été apposés un peu partout par les jeunes, pour s'amuser, ils étaient difficiles à décoller, ils laissaient des traces qu'il fallait nettoyer! Ainsi, les organismes ont dû en cesser la distribution et se sont retrouvés avec des centaines d'autocollants non distribués. L'été suivant, la directrice des EssentiElles se trouvait à un festival de musique lorsqu'elle a aperçu dans le coffre de sa voiture une pleine boîte desdits autocollants. Pour s'en débarrasser, elle a décidé, avec l'aide d'amis, de les distribuer de façon informelle à des festivaliers. La réaction positive qu'elle a obtenue l'a étonnée : elle a reçu plusieurs commentaires sur le fait que c'était une bonne idée de partager ce message sur les lieux du festival, et qu'il s'agissait là d'un moyen original et créatif. C'est ainsi qu'a germé l'idée de la Campagne du consentement, laquelle a été officiellement lancée durant l'été 2011.

Destinée au public des festivals yukonnais, la campagne est basée sur une approche dite "sex positive " qui reconnait que la 
sexualité est fondamentalement une chose positive dont on doit discuter ouvertement (Glickman, 2000). La sex positivity passe par un langage clair et respectueux du corps et de la sexualité et par des interventions non moralisatrices, faisant la promotion de l'appropriation du pouvoir sur sa propre sexualité, et ce, dans le respect des autres. Dans le cadre de la Campagne du consentement, la sex positivity permet de promouvoir des rapports sexuels sécuritaires et égalitaires. En abordant ouvertement le sujet de la sexualité, il est possible de parler sans tabous de la question du consentement en discutant par exemple de trucs concrets pour s'assurer du consentement éclairé venant de la partenaire ou du partenaire.

La campagne telle que je l'ai vécue à l'été 2013 était l'aboutissement de trois ans de développement et d'adaptation du concept. Cet été-là, nous avons été présents dans cinq événements, notamment aux célèbres Atlin Arts and Music Festival et Dawson City Music Festival. À chaque endroit, nous tenions un kiosque, base de nos interventions. Une équipe de bénévoles y était présente ainsi que parmi les festivaliers; elle abordait les gens, les invitant à remplir un questionnaire sur le consentement. Ce dernier comprenait des questions simples de type "vrai ou faux » et servait à engager une conversation sur différents enjeux reliés au consentement et à la violence sexuelle. Les questions avaient été choisies avec soin de façon à mettre à jour des facettes importantes de la problématique, entre autres, le consentement doit être explicite, une personne intoxiquée ne peut pas consentir et consentir à un acte ne veut pas dire consentir à un autre. Le questionnaire abordait également la question des inégalités de genre qui sont à la base de la violence sexuelle.

Une fois le questionnaire rempli, les personnes participantes recevaient un macaron fabriqué à la main et portant un message sur le consentement. Elles étaient également invitées à se rendre au kiosque pour fabriquer leurs propres macarons. Élément assez simple, les macarons constituaient en fait l'élément le plus populaire de la campagne. Arborant des messages ou des dessins amusants et accrocheurs, ils devenaient rapidement une occasion de concours pour trouver les slogans, les rimes ou les illustrations 
les plus originaux sur le thème du consentement. Tout en étant divertissants, les macarons conservaient un important aspect de sensibilisation sur le consentement sexuel. Ils attiraient l'attention par leur créativité et ils permettaient souvent d'amorcer avec les gens une discussion sur le sujet.

Formée sur la violence sexuelle, l'équipe de bénévoles de la campagne était également préparée pour adapter ses interactions avec un public varié en âge. Ainsi, avec les enfants qui aimaient beaucoup fabriquer leurs propres macarons, une discussion simple sur la notion de consentement était entreprise, par exemple, sur des moyens de respecter ses limites et celles des autres. Des messages comme "Non veut dire non " ou "J'aime, donc je respecte " étaient utilisés. L'équipe de la campagne était en effet d'avis qu'il n'est jamais trop tôt pour parler de consentement aux enfants et qu'il y a une façon de le faire qui est appropriée à leur âge et à leur réalité.

Les festivals de musique étant des événements très courus au Yukon, la campagne réussit chaque année à rejoindre un nombre élevé de personnes. Des individus qui fréquentent plus d'un festival nous reconnaissent et reviennent nous parler ou fabriquer un nouveau macaron. Grâce à cette présence aux événements culturels qui constituent d'importants points de rassemblement pour la communauté, la Campagne du consentement et son équipe sont bien connues sur le territoire.

\section{Des défis importants, des résultats inspirants}

Comme pour la plupart des initiatives de prévention de la violence, il est difficile de mesurer de façon précise les impacts de la campagne. Cependant, par le nombre élevé de conversations engagées avec le public et par la teneur de certaines discussions, on constate des résultats positifs. Il n'est pas rare pour les travailleuses, travailleurs et bénévoles de la campagne de voir leurs interactions se transformer avec des groupes de festivaliers en débats amicaux, et animés, sur différentes questions : est-ce que les femmes sont en quelque sorte responsables d'être agressées si elles s'habillent 
d'une certaine manière? Quelles sont les causes des agressions sexuelles? Plusieurs personnes se voient étonnées de découvrir certaines statistiques sur la violence sexuelle au Canada et au Yukon. Ainsi, on peut voir que la campagne remplit un objectif de sensibilisation et de façon encore plus significative, qu'elle parvient à susciter des questionnements, des remises en question et des prises de conscience sur des enjeux essentiels reliés à cette problématique. Il s'agit là d'étapes importantes dans la prévention de la violence.

En outre, selon moi, un des succès principaux de la Campagne du consentement demeure dans son efficacité à rejoindre un public qui autrement ne s'informerait pas nécessairement sur ses enjeux. On voit, au Yukon comme ailleurs, une panoplie d'événements organisés sur le sujet de la violence faite aux femmes. Cependant, on remarque que souvent ce sont des personnes déjà conscientisées sur le sujet qui s'y intéressent. En abordant le large public des festivals, la campagne réussit à rejoindre une diversité d'individus, allant de personnes très informées, qui ont déjà réfléchi à la question, à d'autres qui n'en connaissent presque rien. De plus, le succès de la campagne se reflète dans la réponse en grande majorité positive du public. En effet, il est rare que les gens, toujours abordés de façon respectueuse et dynamique, refusent de participer au questionnaire ou de parler à ses bénévoles. Aussi, l'équipe reçoit souvent des commentaires enthousiastes sur son travail et elle rencontre même beaucoup de personnes qui expriment leur reconnaissance d'avoir pu y participer.

Un autre aspect positif de la campagne se trouve dans l'implication variée au sein de la communauté et la collaboration entre plusieurs acteurs. En plus d'être bâtie sur une coopération entre les collectivités féministes anglophones, autochtones et francophones, comme mentionné plus haut, la campagne suscite la collaboration des organisations des festivals avec ses propres bénévoles qui reçoivent une formation au début de l'été ainsi qu'un suivi au fil des festivals et qui deviennent des agentes et agents de la prévention de la violence sexuelle. Plusieurs décident de s'impliquer dans la campagne pour plus d'un été et poursuivent même leur implication dans d'autres projets durant 
l'année. Par contre, depuis les débuts, un défi se pose en ce qui concerne les bénévoles, soit la quasi-absence parmi eux de représentantes ou de représentants des Premières Nations (un ou deux par été, tout au plus). L'organisation de la campagne essaie toujours de résoudre le problème et d'y assurer une représentativité de la population autochtone.

Les interactions lors de la campagne offrent des résultats intéressants, mais présentent aussi des limites importantes. De fait, il est difficile dans le contexte de divertissement des festivals d'aborder en profondeur des sujets aussi complexes que les inégalités de genre et la culture du viol. Les sujets ne sont souvent qu'effleurés avec les festivaliers. De plus, un défi réside dans la résistance de certaines personnes à remettre en question leurs préjugés en matière d'agressions sexuelles. Encore nombreux sont les répondantes ou répondants qui considèrent par exemple que la violence sexuelle est le fait de certains comportements d'individus isolés plutôt qu'une problématique sociale ancrée dans des inégalités entre hommes et femmes. Alors que la plupart de ces individus acceptent tout de même de discuter du sujet, d'autres se renferment sur leurs positions et refusent toute ouverture dans leur conversation avec l'équipe de la campagne. Une petite proportion de gens refuse totalement toute interaction à ce sujet, ou alors remplit le questionnaire de façon distraite, sans souhaiter en approfondir les enjeux qu'il soulève. Les résultats sont alors très limités auprès de ces personnes.

Malgré ses défis, la Campagne du consentement constitue un modèle d'intervention qui mérite d'être exploité dans d'autres milieux. Mon implication dans la campagne m'a permis de développer des habiletés à informer sur la violence sexuelle et d'en discuter de façon dynamique avec le public afin de susciter des questionnements, des débats et des prises de conscience. J'ai constaté que l'approche sex positive permet d'aborder la question de la sexualité dans toute sa complexité, en mettant l'accent sur l'importance des rapports égalitaires et sécuritaires. L'implication de la minorité francophone dans la lutte contre la violence faite aux femmes auYukon est un exemple inspirant d'une collaboration diversifiée qui permet à des femmes de différents horizons d'être 
représentées dans les actions communautaires qui les concernent en tant que femmes.

\section{Bibliographie}

ASSOCIATION FRANCO-YUKONNAISE (2014). Historique de l'Association franco-yukonnaise, réf. du 5 septembre 2014,

http://www.afy.yk.ca/secteurs/main/fr/index.php?location=m1001-historique.

GLICKMAN, Charlie (2000). "The language of sex positivity », Electronic Journal of Human Sexuality, Vol. 3, réf. du 25 août 2014, www.ejhs.org/volume3/sexpositive.htm

GOUVERNEMENT DUYUKON (2008). Population, réf. du 5 septembre 2014, http://www.gov.yk.ca/fr/facts/population.html.

STATISTIQUE CANADA (2008). Les agressions sexuelles au Canada, Ottawa, Gouvernement du Canada, Centre canadien de la statistique juridique, $21 \mathrm{p}$.

STATISTIQUE CANADA (2012). Série "Perspective géographique ", Recensement de 2011, réf. du 5 septembre 2014,

http://www12.statcan.gc.ca/census-recensement/2011/as-sa/fogs-spg/Facts-pr-fra. cfm?Lang $=$ Fra\&GC $=60$.

STATISTIQUE CANADA (2013). Mesure de la violence faite aux femmes : tendances statistiques, Ottawa, Gouvernement du Canada, $130 \mathrm{p}$.

YUKON BUREAU OF STATISTICS (2012). Population Report - March 2012, Whitehorse, Gouvernement du Yukon, 4 p. 